\title{
All-Optical 2R Regeneration using the Hysteresis in a Distributed Feedback Laser Diode
}

\author{
Koen Huybrechts, Student Member, IEEE, Takuo Tanemura, Member, IEEE, Koji Takeda, Student Member, IEEE, \\ Yoshiaki Nakano, Member, IEEE, Roel Baets, Fellow, IEEE, and Geert Morthier, Senior Member, IEEE
}

\begin{abstract}
A broadband optical $2 \mathrm{R}$ regenerator based on a single distributed feedback laser is demonstrated for NRZ signals at a bitrate of $10 \mathrm{~Gb} / \mathrm{s}$. A semi-analytical approach for the influence of hysteresis on the transfer function of a $2 R$ regenerator is shown.
\end{abstract}

Index Terms $-2 \mathrm{R}$ regeneration, DFB laser, hysteresis.

\section{INTRODUCTION}

$\mathbf{T}$ HE telecommunication industry has experienced a huge growth in the last years and the need for bandwidth is expected to increase further as new internet-based services are being implemented. Optical point-to-point wavelengthdivision-multiplexed (WDM) network links were able to fulfill the capacity requirements in the past and have been implemented world-wide. However, to meet the future demand, the logical next step in optical network evolution will be to implement the routing and switching in the optical domain. A major concern is however the accumulation of noise which severly limits the cascadability of optical network nodes. Different techniques for $2 \mathrm{R}$ regeneration have been proposed in the past such as devices based on interferometers [1] and self-phase modulation [2], [3].

Recently, we demonstrated that a distributed feedback (DFB) laser diode shows a hysteresis in the lasing power under the injection of a holding beam [4], [5]. The underlying effect for the bistability is the spatial hole burning induced nonlinearity. The bistability at the lasing wavelength has been successfully applied to obtain flip-flop operation [4] and all-optical packet switching [6]. However, as illustrated in Figure 1, the bistability is not only present at the lasing wavelength, but also in the transmission characteristics of the injected light. In this article, we will demonstrate with numerical and experimental results that the transmission characteristic of the injected light can be used to improve the bit-error rate and is therefore applicable for $2 \mathrm{R}$ regeneration in optical systems. The injected light can have any wavelength outside the stopband of the DFB

Manuscript received September 1, 2009;

This work is financed by the Fund for Scientific Research (FWO), the IAP-project "Photonics@be" and the Erasmus Mundus program. The work of K. Huybrechts is supported by the Institute for the Promotion of Innovation through Science and Technology in Flanders (IWT) under a specialization grant.

K. Huybrechts, R. Baets and G. Morthier are with the Photonics Research Group, Department of Information Technology (INTEC), Ghent University - IMEC, Sint-Pietersnieuwstraat 41, B-9000 Ghent, Belgium (author e-mail: Koen.Huybrechts@intec.UGent.be)

T. Tanemura, K. Takeda and Y. Nakano are with the Research Center for Advanced Science and Technology (RCAST), University of Tokyo, 4-6-1 Komaba, Meguro-ku, Tokyo, 153-8904, Japan grating resulting in broadband operation of the $2 \mathrm{R}$ regenerator. This is a major advantage over $2 \mathrm{R}$ regenerators based on four wave mixing [7] and injection-locking [8], [9].

To the authors' knowledge, no research has been performed yet on the influence of hysteresis for $2 \mathrm{R}$ regeneration. In [10], [11], a theoretical approach is given for hysteresisbased detectors in networks to give a sub-optimal solution for the mismatch between the fixed bandwidth of electrical filters and the different bit-rates present in an optical network. The experimental results that will be presented in this article suggest that the hysteresis in the transmission characteristic plays an important role and we will give the basis for a theoretical analysis on $2 \mathrm{R}$ regeneration with a hysteresis in the next section. Intuitively, one can consider that instead of a fixed decision level for the zero's and ones, the hysteresis causes the decision level to change dynamically and therefore reduces the bit error rate of a noisy signal. As outlined in [3], [12], distinctive transfer functions for the ones and zeros are necessary for improvement of the bit-error rate (BER) of a signal. We follow a more intuitive approach but the presence of a hysteresis suggests that this condition is fulfilled and that our regenerator is what is called a class II optical regenerator in [3].

\section{THEORETICAL APPROACH}

In this section, we will investigate the influence of a hysteresis in the transmission characteristic of a $2 \mathrm{R}$ regenerator. The concept is illustrated in Figure 2. We assume a step function (Figure 2a) with a hysteresis of width $h$. When a noisy signal is sent through the regenerator, a normal regenerator will distinguish between the ones and zeros using a static decision level. We will assume that the probability distribution function of noise on the one $\left(\mathrm{pdf}_{1}\right)$ and zero $\left(\mathrm{pdf}_{0}\right)$ levels is equal and the optimal position of the decision characteristic is at $1 / 2$. We also assume that the electrical signal-to-noise ratio after the receiver is predominantly determined by the optical signal to noise ratio before the receiver.

When we use a hysteresis in the transfer characteristic on the other hand, the decision level will change dynamically with the bit pattern. This will improve the bit-error rate because the transfer function will be different for the ones and zeros [12]. As can be seen in Figure 2(c), the decision level will be lower for a one and higher for a zero allowing a wider distribution of noise. There is a small adverse effect caused by the increased threshold to change from one state to the other, but we will demonstrate that for small values of the hysteresis and fast regenerators this has little or no influence. 



Fig. 1. Bistability in a DFB laser diode under injection of light at a different wavelength. (a) Schematic representation of a $\lambda / 4$ DFB laser; (b) Simulation of transmission of light at a different wavelength through a DFB laser (b) The corresponding laser output power.

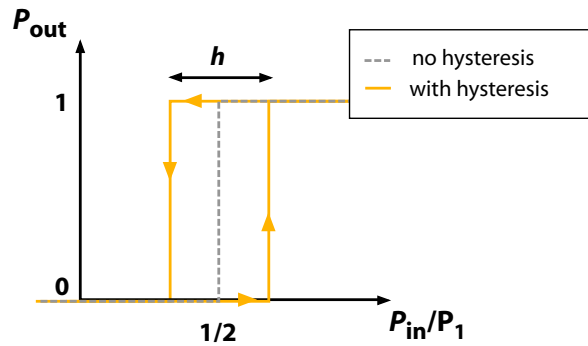

(a)
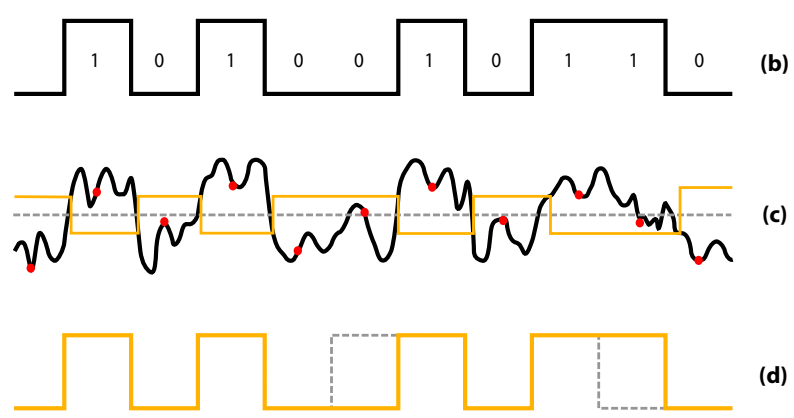

(d)

Fig. 2. Concept of using a hysteresis for $2 R$ regeneration (a) Transmission characteristic of the regenerator; (b) original bit-pattern; (c) bit-pattern with noise with a dynamically fluctuating decision level (orange) and static decision level (dashed); (d) reconstructed bit pattern at the detector after regeneration with hysteresis (orange) and without (dashed).

The textbook definition [13] for the BER is given in terms of the probability $P(0 \mid 1)$ of deciding zero when a one is expected and the probability $P(1 \mid 0)$ of deciding one when a zero is expected. Since 1 and 0 bits are equally likely to occur, the BER can be defined as:

$$
\mathrm{BER}=\frac{1}{2}(P(0 \mid 1)+P(1 \mid 0))
$$

In case of standard Gaussian noise, the bit-error rate can be derived as a function of the $Q$ parameter [13] by:

$$
\begin{aligned}
\text { BER } & =\frac{1}{2} \int_{-\infty}^{1 / 2} \operatorname{pdf}_{1}(P) d P+\frac{1}{2} \int_{1 / 2}^{\infty} \operatorname{pdf}_{0}(P) d P \\
& =\frac{1}{2} \operatorname{erfc}\left(\frac{Q}{\sqrt{2}}\right)
\end{aligned}
$$

where we assumed that the noise distribution on the ones and zeros is the same. The $Q$-parameter in the above equation is defined as the ratio between signal power and the standard deviation of the Gaussian noise distribution [13].

When a decision characteristic with a hysteresis is used (as in Figure 2a), we should consider a more complex analysis where the output depends on the state in a previous time step. This means that we will need to reconstruct the analysis to show how the device will switch states during a single bit period. The noise on these signals is mainly coming from ASE (amplified spontaneous emission) noise of the amplifiers and therefore fluctuating very fast. If we assume that the device has a reaction time that is significantly shorter than the duration of a single bit, we can look at it from the point of view of the threshold level which will change at the beginning of a bit period following a transition in the bit sequence while the noise is changing throughout the bit period. We will follow a similar procedure as in [11] and divide the time window of one bit in $N$ different time steps. The error probability when there is no state difference will be lower than considered with a standard fixed decision characteristic. There is however also an adverse effect because of the higher threshold of changing from a zero bit to a one bit and vice versa. In the following analysis, we will show that this adverse effect can be overcome when the regenerator acts faster than the duration of individual bits and when the hysteresis is not very wide.

We can write the probability $p_{x y \mid z}$ to change from state $x$ 
to $y$ while $z$ is given, as follows:

$$
\begin{aligned}
& p_{00 \mid 1}=\int_{-\infty}^{(1+h) / 2} \operatorname{pdf}_{1} d P=\frac{1}{2} \operatorname{erfc}\left((1-h) \frac{Q}{\sqrt{2}}\right) \\
& p_{10 \mid 1}=\int_{-\infty}^{(1-h) / 2} \operatorname{pdf}_{1} d P=\frac{1}{2} \operatorname{erfc}\left((1+h) \frac{Q}{\sqrt{2}}\right) \\
& p_{01 \mid 0}=\int_{(1+h) / 2}^{\infty} \operatorname{pdf}_{0} d P=\frac{1}{2} \operatorname{erfc}\left((1+h) \frac{Q}{\sqrt{2}}\right) \\
& p_{11 \mid 0}=\int_{(1-h) / 2}^{\infty} \operatorname{pdf}_{0} d P=\frac{1}{2} \operatorname{erfc}\left((1-h) \frac{Q}{\sqrt{2}}\right)
\end{aligned}
$$

When using a hysteresis, the previous state is used to determine the next state and a recursive expression for $P_{n}(0 \mid 1)$ (being the probability on time step $n$ to decide zero when a one is expected) can be written as:

$$
\begin{aligned}
P_{n+1}(0 \mid 1) & =P_{n}(0 \mid 1) p_{00 \mid 1}+P_{n}(1 \mid 1) p_{10 \mid 1} \\
P_{1}(0 \mid 1) & =\frac{1}{2} p_{00 \mid 1}+\frac{1}{2} p_{10 \mid 1}
\end{aligned}
$$

and a similar equation for $P(1 \mid 0)$. Because the noise distribution on the ones is assumed similar to the noise distribution on the zeros $\left(p_{00 \mid 1}=p_{11 \mid 0}\right.$ and $\left.p_{10 \mid 1}=p_{01 \mid 0}\right)$ and $P(1 \mid 1)=1-P(0 \mid 1)$ we can write down the following recursive expression for the BER:

$$
\begin{aligned}
\operatorname{BER}(n+1) & =\operatorname{BER}(n) p_{00 \mid 1}+[1-\operatorname{BER}(n)] p_{10 \mid 1} \\
\operatorname{BER}(1) & =\frac{1}{2} p_{00 \mid 1}+\frac{1}{2} p_{10 \mid 1}
\end{aligned}
$$

The second term of Equation 4 appears to be dominant for realistic values of the hysteresis $(h<1)$ and the bit error rate asymptotically becomes equal to $p_{10 \mid 1}$ after a sufficient amount of time steps. This asymptotical bit error rate is depicted in Figure 3 for different values of the hysteresis width. However, this figure does not take into account the adverse effect of the higher threshold for switching between two different states. As discussed above, the bit error rate will improve during the time window of a single bit. For high values of the hysteresis width, it will take more time steps to achieve an improvement. The evolution of the BER is depicted in Figure 4 for different time steps $n$ and a hysteresis width $h=0.4$. We can observe an improvement in bit error rate after 3 time steps. This means that - according to this model - the reaction time of the device should be 4 or 5 times faster than the bit duration when $h=$ 0.4 . The number of time steps needed for an improvement in bit error rate increases with the hysteresis width, eg. for a hysteresis of $h=0.1$ there is already an improvement in the second time step but it takes 15 time steps for a hysteresis width of $h=0.9$.

We can conclude this section by stating that a hysteresis in the transmission characteristic makes the decision threshold to move dynamically with the signal. Therefore, a distinctive transfer function for the ones and zeros allows to improve the bit error rate. The theoretical model that is derived above, suggests however that the response time of the regenerator should be significantly faster with respect to the bitrate of the original signal.



Fig. 3. Asymptotical behaviour of BER as a function of the $Q$ parameter for different values of the hysteresis width $h$ (and $n \rightarrow \infty$ ).



Fig. 4. BER as a function of the $Q$ parameter at different time steps $n$ for a hysteresis with width $h=0.4$. The dashed line represents the situation without hysteresis.

\section{EXPERIMENT}

For the experiment, we use the set-up as depicted in Figure 5. A pulse pattern generator (PPG) generates a pseudorandom bit-sequence (PRBS) of $2^{31}-1$ bits. The original signal is being attenuated and amplified to decrease the OSNR. The regenerator is a standard, non-optimized $\lambda / 4$-shifted DFB laser diode from Alcatel-Thales with AR-coated facets. It has a $\kappa L$ value of 1.6 , a bias current of $150 \mathrm{~mA}$ and its central wavelength is $1553 \mathrm{~nm}$. Lensed fibers are aligned at both sides of the laser to couple the light. The degenerated signal has an input power of 5-7 $\mathrm{dBm}$ (depending on the power injected in the EDFA) and is combined with a holding beam of $5 \mathrm{dBm}$ (both measured in fiber after the coupler). The holding beam is used to decrease the threshold for bistability and can be adjusted by an attenuator. The width of the bistability is approximately $1 \mathrm{~dB}$ wide (resulting in a value for $h$ between 0.1 and 0.2 in the analysis of section II). An optical band- 


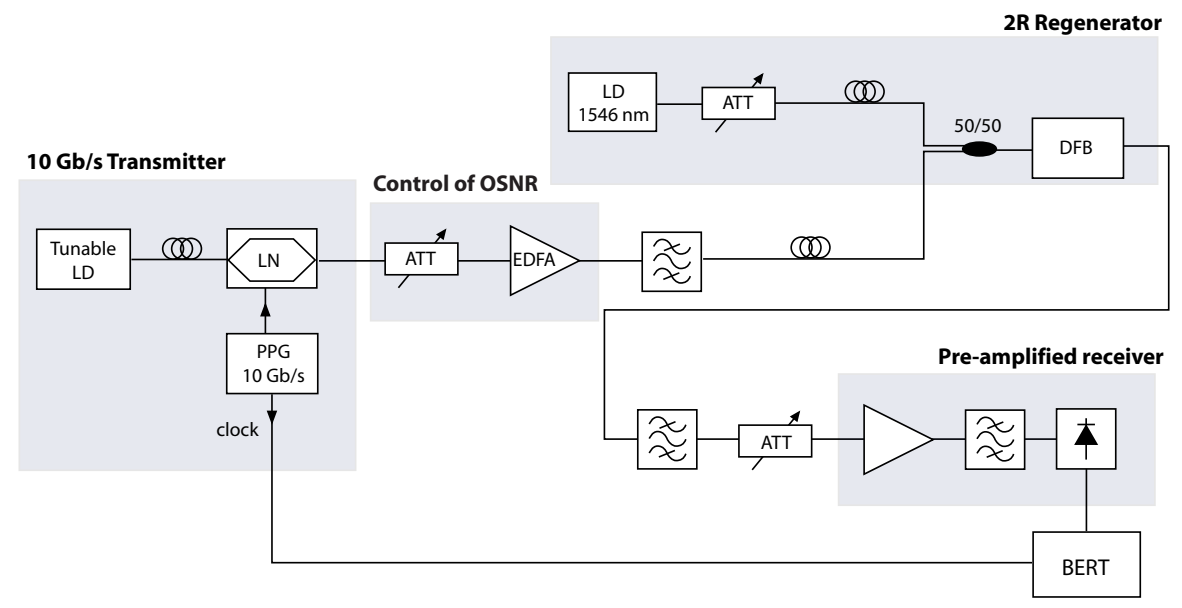

Fig. 5. Schematic of the set-up. (LD: laser diode; LN: lithium-niobate modulator; PPG: pulse pattern generator; ATT: attenuator; EDFA: erbium-doped fiber-amplifier; BERT: bit error rate tester.

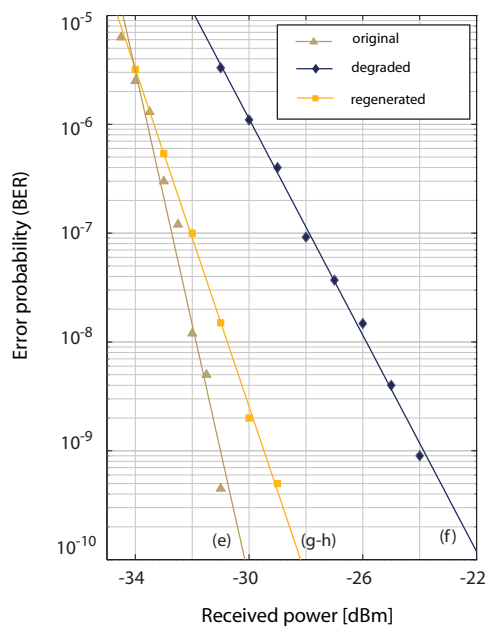

(a)

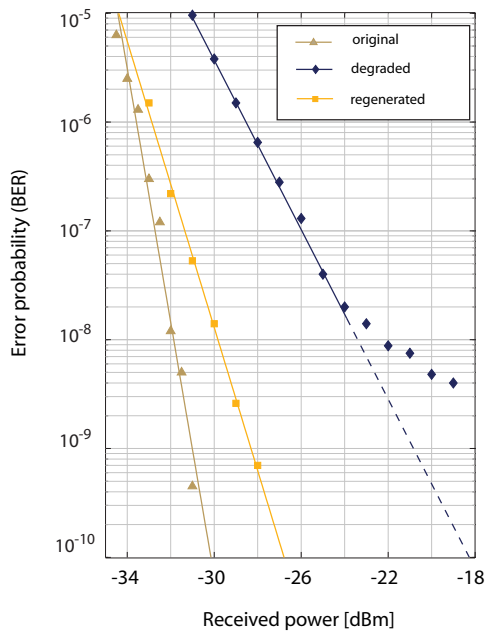

(b)

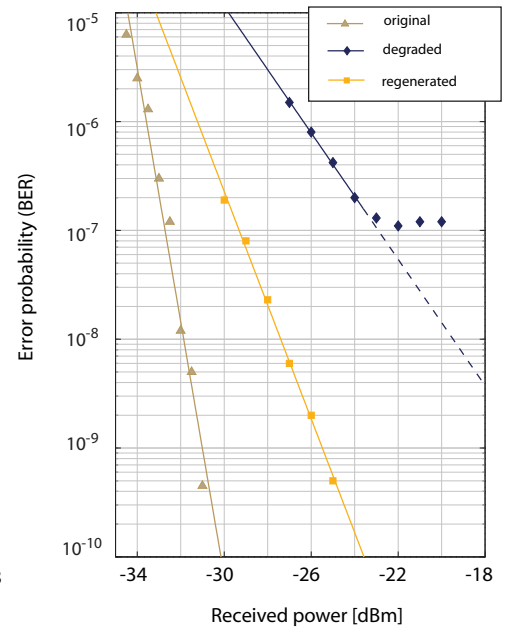

(c)

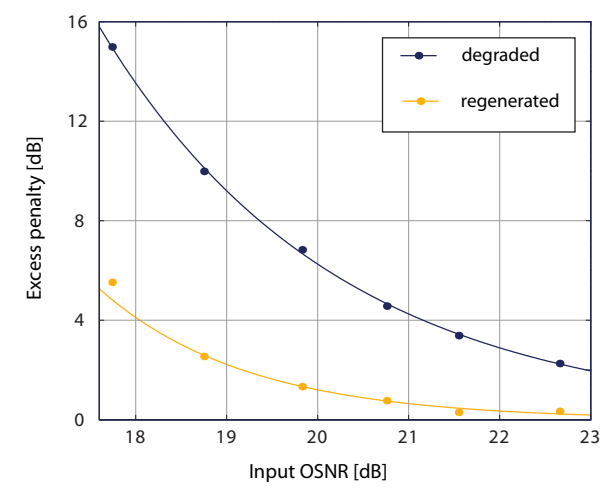

(d)

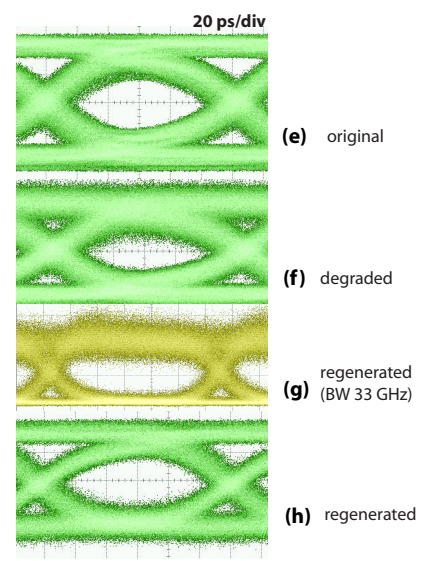

Fig. 6. BER as a function of the received optical power for different values of the OSNR, the power excess diagram and corresponding eye diagrams. (a) Input OSNR of $19.83 \mathrm{~dB}$; (b) Input OSNR of $18.75 \mathrm{~dB}$; (c) Input OSNR of $17.74 \mathrm{~dB}$; (d) Excess penalty for a BER of $10^{-9}$ of the degraded and regenerated signal compared to the original signal as a function of the input OSNR; (e) eye diagram of original signal from (a) at the receiver; (f) eye diagram of degraded signal; $(\mathrm{g})$ eye diagram of the regenerated signal without electric filter (receiver bandwidth of $33 \mathrm{GHz}$ ); (h) eye diagram of regenerated signal. 
pass filter with a width of $1 \mathrm{~nm}$ removes the lasing light so that only the signal at the original wavelength is sent to the pre-amplified receiver for a bit-error rate (BER) analysis. A variable attenuator is used to change the received optical power on the receiver in order to make BER-diagrams. $\mathrm{F}$

In Figure 6a-c, the BER diagrams as a function of the received optical power are shown for different values of the EDFA input power (and thus for different OSNR's). From these diagrams, it is clear that the $2 \mathrm{R}$ regenerator is able to improve the degraded signal significantly. Its noise suppression capabilities are demonstrated by Figure $6 \mathrm{~d}$ where the excess power penalty for the regenerated and degraded signal compared to the original signal are depicted as a function of the EDFA input power. The corresponding eye diagrams for the regeneration are depicted in Figure 6e-h. In Figure 6g, the regenerated signal is shown without an electrical filter that is matched with the $10 \mathrm{GHz}$ bandwidth at the receiver side but on a regular optical scope with a $33 \mathrm{GHz}$ bandwidth. This eye diagram illustrates that the regenerator might work also at higher bitrates. We want to point out that our $2 \mathrm{R}$ regenerator with hysteresis even reduces the BER when the degraded signal reaches the noise floor, determined by the OSNR; something which is beyond the capabilities of regenerators with a single decision level.

The extinction ratio improvement is depicted in Figure 7. However, in this diagram, we did not take the influence of the $5 \mathrm{dBm}$ holding beam into account and an amplified signal without holding beam might result in a better extinction ratio improvement. In Figure 8, the OSNR at the output of the $2 \mathrm{R}$ regenerator is depicted as a function of the OSNR at the input. The OSNR is measured by setting the resolution of the spectrum analyzer to $0.1 \mathrm{~nm}$ and determining the difference between the signal and noise. The reduction of noise is important with regard to the cascadability of the regenerators.

The $2 \mathrm{R}$ regenerator can work at any wavelength outside the stopband of the DFB grating as illustrated for three arbitrary wavelengths in Figure 9. The BER diagrams for different injected wavelengths are depicted and show broadband operation that is only limited by the spectral width of the gain medium. The gain medium in our device are quantum wells leading to a gain bandwidth of approximately $20 \mathrm{~nm}$. The small differences between the graphs are mostly due to the spectral variation in gain of the EDFA.

When we separate the signal at the lasing wavelength (instead of the injected wavelength), we obtain the reversed, wavelength converted signal. The noise floor is much higher and error-free wavelength conversion was only obtained at a smaller bitrate of $3 \mathrm{~Gb} / \mathrm{s}$. This is because the carrier density changes much faster than the laser signal which also depends on the cavity decay time.

\section{CONCLUSION}

A new concept for $2 \mathrm{R}$ regeneration is proposed using a hysteresis in the transmission characteristic. The concept is illustrated with experimental results showing bit error rate improvement of a $10 \mathrm{~GB} / \mathrm{s} \mathrm{NRZ}$ signal using a single distributed

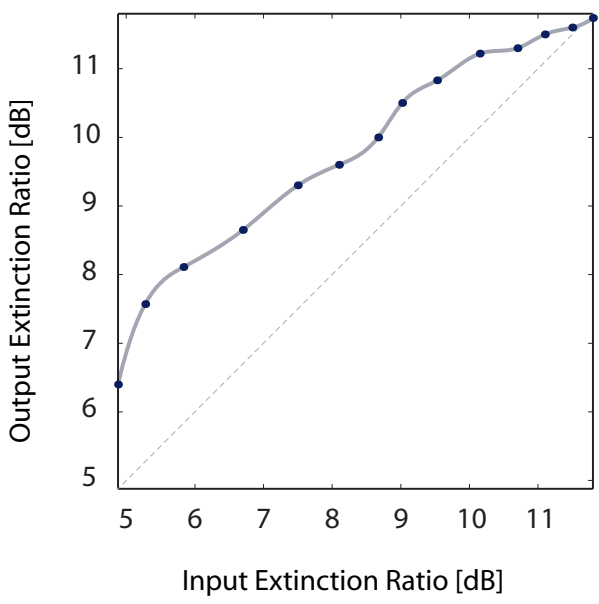

Fig. 7. The extinction ratio improvement.

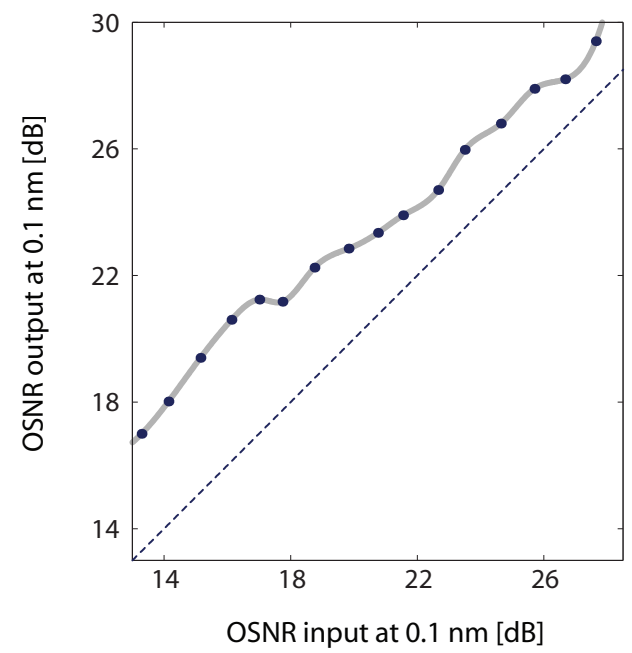

Fig. 8. OSNR in $0.1 \mathrm{~nm}$ at the output of the all-optical $2 \mathrm{R}$ regenerator as a function of the input OSNR.

feedback (DFB) laser diode. The simplicity of the concept and the good performance make this regenerator suitable for application in optical access or metro networks. Since direct modulation of DFB lasers has been demonstrated at higher bitrates (up to $40 \mathrm{GHz}$ ), the presented technique might be also employed at higher speeds by using optimalized designs.

\section{ACKNOWLEDGMENT}

The authors would like to thank Guang-Hua Duan from Alcatel-Thales III/V-labs for providing the DFB laser diodes.

\section{REFERENCES}

[1] D. Wolfson, A. Kloch, T. Fjelde, C. Janz, B. Dagens, and M. Renaud, "40-Gb/s all-optical wavelength conversion, regeneration, and demultiplexing in an soa-based all-active mach-zehnder interferometer," IEEE Photonics Technology Letters, vol. 12, no. 3, pp. 332-334, 2000.

[2] P. V. Mamyshev and T. E. T. E. Telefon Espana, "All-optical data regeneration based on self-phase modulation effect," in 24th European Conference on Optical Communications. Madrid, Spain: Telefonica Espana Sa, 1998, pp. 475-476. 

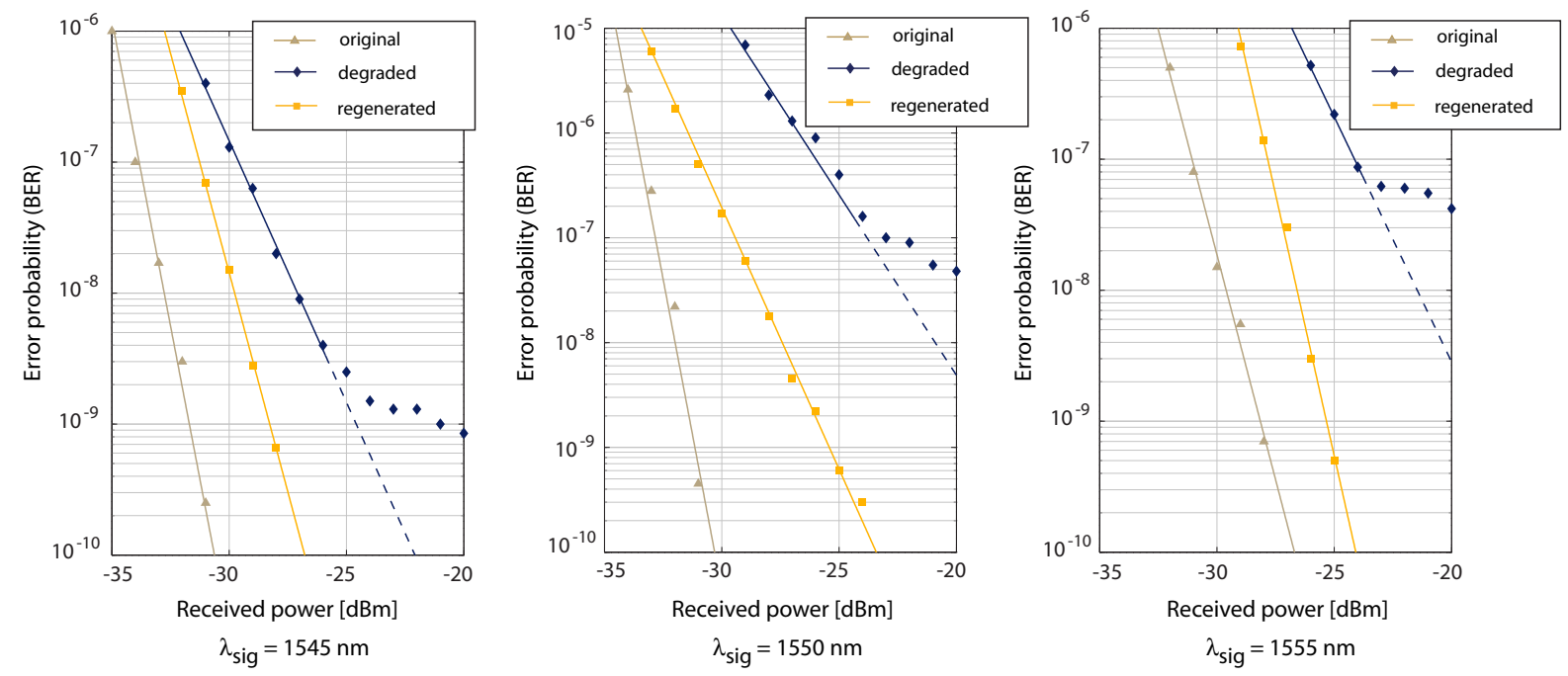

Fig. 9. Wavelength independence: BER as a function of the received optical power for different transmission wavelengths.

[3] M. Rochette, L. B. Fu, V. Ta'eed, D. J. Moss, and B. J. Eggleton, "2R optical regeneration: An all-optical solution for BER improvement," IEEE Journal of Selected Topics in Quantum Electronics, vol. 12, no. 4, pp. 736-744, 2006.

[4] K. Huybrechts, G. Morthier, and R. Baets, "Fast all-optical flip-flop based on a single distributed feedback laser diode," Optics Express, vol. 16, no. 15, pp. 11405-11410, 2008.

[5] K. Huybrechts, W. D'Oosterlinck, G. Morthier, and R. Baets, "Proposal for an all-optical flip-flop using a single distributed feedback laser diode," Photonics Technology Letters, vol. 20, no. 1-4, pp. 18-20, 2008.

[6] K. Huybrechts, T. Tanemura, Y. Nakano, R. Baets, and G. Morthier, "40-Gb/s all-optical packet switching with a distributed-feedback laser as all-optical flip-flop," IEEE Photonics Technology Letters, vol. 21, no. 9-12, pp. 703-705, 2009.

[7] H. Simos, A. Bogris, and D. Syvridis, "Investigation of a 2R all-optical regenerator based on four-wave mixing in a semiconductor optical amplifier," Journal of Lightwave Technology, vol. 22, no. 2, pp. 595-604, 2004.

[8] A. Kuramoto and S. Yamashita, "All-optical regeneration using a sidemode injection-locked semiconductor laser," Selected Topics in Quantum Electronics, IEEE Journal of, vol. 9, no. 5, pp. 1283-1287, 2003.

[9] S. Yamashita and J. Suzuki, "All-optical 2R regeneration using a two-mode injection-locked fabry-perot laser diode," IEEE Photonics Technology Letters, vol. 16, no. 4, pp. 1176-1178, 2004.

[10] C. E. Korman, R. Barry, and U. C. Kozat, "On binary detection with hysteresis," Siam Journal on Applied Mathematics, vol. 62, no. 5, pp. 1794-1809, 2002.

[11] C. E. Korman, "A discrete-time model for binary detection with rectangular hysteresis operators," in 5th International Symposium on Hysteresis and Micromagnetic Modeling (HMM 2005). Budapest (Hungary): Elsevier Science Bv, 2005, pp. 17-20.

[12] M. Rochette, J. N. Kutz, J. L. Blows, D. Moss, J. T. Mok, and B. J. Eggleton, "Bit-error-ratio improvement with $2 \mathrm{R}$ optical regenerators," IEEE Photonics Technology Letters, vol. 17, no. 4, pp. 908-910, 2005.

[13] G. Agrawal, Fiber-optic communication systems. John Wiley and sons, pp. $162-164$

Koen Huybrechts (S'06) received his master degree in Physics Engineering from Ghent University in 2006. During his masters, he spent one year as an exchange student at KTH in Stockholm. Currently, he is working towards a $\mathrm{PhD}$ degree at the Photonics Research Group of Ghent University - IMEC. His research includes mainly all-optical flip-flops and all-optical signal processing devices. He is vice-chairman of the IEEE Photonics Society Benelux Student Chapter.
Takuo Tanemura (S'02M'06) received the B.E., M.S., and Ph.D. degrees in electronic engineering, all from the University of Tokyo, Japan, in 2001, 2003. and 2006, respectively. He joined the Department of Electronic Engineering, University of Tokyo in 2006 and moved to the Research Center for Advanced Science and Technology, University of Tokyo in 2007, where he is currently a Lecturer. His current research interest includes photonic integrated circuits based on III-V semiconductors, photonic switching networks, and all-optical signal processing devices. Dr. Tanemura is a member of IEEE Lasers and Electro-Optics Society (LEOS) and the Institute of Electronics, Information, and Communication Engineers (IEICE) of Japan. He is the recipient of the 2005 IEEE LEOS Graduate Student Award and the 2006 Ericsson Young Scientist Award. He has authored and coauthored over 20 refereed journal publications and over 50 international conference papers.

Koji Takeda (S'06) was born in Chiba, Japan, in 1982. He received the B.E. and M.E. degrees in electronic engineering, all from the University of Tokyo, Tokyo, Japan, in 2005 and in 2007, respectively. He has belonged to school of engineering, the University of Tokyo as a Ph.D candidate. He is also with the Research Center for Advanced Science and Technology, the University of Tokyo. He has received the Research Fellowship for Young Scientists from Japan Society for the Promotion of Science (JSPS) since 2007. In 2008, he spent two weeks at the University of Rochester, New York, under the cooperative program of the JSPS. His research has mainly focused on semiconductor photonic integrated circuits based on III-V materials, especially on all-optical flip-flops and switches. Mr. Takeda is a student member of IEEE/LEOS and the Japan Society of Applied Physics (JSAP).

Yoshiaki Nakano (S'81M'87) received the B.E., M.S., and Ph.D. degrees in electronic engineering from the University of Tokyo, Tokyo, Japan, in 1982, 1984 , and 1987, respectively. In 1984, he spent a year at the University of California, Berkeley, as an exchange student. 
In 1987, he joined the Department of Electronic Engineering, University of Tokyo, where he was an Associate Professor in 1992, a Professor in 2000, and the Department Head in 2001, and is currently the Vice Director and a Professor in the Research Center for Advanced Science and Technology, and is also with the Department of Electronic Engineering, School of Engineering. In 1992, he was a visiting Associate Professor at the University of California, Santa Barbara. He was the Project Leader of the Japanese National Project on Photonic Networking Technology organized by the Ministry of Economy, Trading, and Industry, and as the Project Leader of the Solution Oriented Research for Science and Technology (SORST) Program on Non-reciprocal Semiconductor Digital Photonic Integrated Circuits and their Applications to Photonic Networking sponsored by the Japan Science and Technology Corporation. He authored and coauthored over 200 refereed journal publications and over 400 international conference papers, and holds 40 patents. He was the Editor-in-Chief of the Applied Physics Express (APEX) and Japanese Journa of Applied Physics (JJAP). His current research interests include physics and fabrication technologies of semiconductor distributed feedback lasers, semiconductor optical modulators/switches, and monolithically integrated photonic circuits.

Prof. Nakano was an elected member of the Board of Governors of the IEEE Laser Electro-Optical Society (LEOS, nowthe Photonics Society) and a member of the Board of Directors of the Japan Society of Applied Physics (JSAP). He is a member of the Board of Directors of the Japan Institute of Electronics Packaging (JIEP), the Chairman of the Optoelectronics Technology Trend Research Committee of the Optoelectronics Industry and Technology Development Association (OITDA), and the Chairman of the Optical Interconnect Standardization Committee of Japan Electronics Packaging and Circuits Association. He is also a Fellow of the Institute of Electronics, Information, and Communication Engineers (IEICE) and a member of the IEEE Electron Devices Society and the Optical Society of America. He is the recipient of the 1987 Shinohara Memorial Prize from the IEICE, the 1991 Optics Paper Award from the JSAP, the 1997 Marubun Science Prize, the 2007 Ichimura Prize, the 2007 IEICE Electronics Society Award, and the 2007 Sakurai Medal from the OITDA. He was presented the Prime Minister Award in Collaborative Research between Academia and Industry in 2007.

Roel Baets (M'88, SM'96) received the degree in electrical engineering from Ghent University, Belgium, in 1980. He received the M.Sc. degree in electrical engineering from Stanford University in 1981 and the Ph.D. degree from Ghent University in 1984. Since 1981 he has been with the Department of Information Technology (INTEC) of Ghent University. Since 1989 he is a professor in the engineering faculty of Ghent University. From 1990 till 1994 he has also been a part-time professor at the Technical University of Delft, The Netherlands. Since 2004 he is also part-time professor at the Technical University of Eindhoven.

Roel Baets has mainly worked in the field of photonic components. With about 250 journal publications and 500 conference papers as well as about 15 patents he has made contributions to research on semiconductor laser diodes, passive guided wave and grating devices and to the design and fabrication of photonic ICs, both in III-V semiconductors and in silicon. He leads the Photonics Research Group at Ghent University-INTEC (associated lab of IMEC), which focuses on new concepts for photonic components and circuits for optical communication, optical interconnect and optical sensing. He has been involved in various European research projects and has been coordinator of some of them. Currently he coordinates the European Network of Excellence ePIXnet.

Roel Baets is a member of the Optical Society of America, IEEE-LEOS, SPIE and the Flemish Engineers Association. He has been member of the program committees of a.o. OFC, ECOC, IEEE Semiconductor Laser Conference, ESSDERC, CLEO-Europe, LEOS Annual Meeting, Photonics Europe and ECIO. He has been chairman of the IEEE-LEOS-Benelux chapter from 1999 to 2001. From 2003 to 2005 he was an elected member of the Board of Governors of IEEE-LEOS. He is a Fellow of the IEEE.

Geert Morthier (M'93, SM'01) received the degree in electrical engineering and the Ph.D. degree from the University of Gent in 1987, 1991, respectively. In 2001 he was appointed partime professor at Ghent University.
Since 1991 he is a member of the permanent staff of IMEC. His main interests are in the modelling and characterisation of optoelectronic components. He has authored or co-authored around 100 papers in the field. He is also one of the two authors of the Handbook of Distributed Feedback Laser (Artech House, 1997) and co-editor of the book How to model and measure photonic components: experience from a European project (Springer-Verlag, 1998). He has been the project manager of the ACTS project ACTUAL dealing with the control of widely tunable laser diodes and of the FP5 IST project NEWTON on new widely tunable lasers. He is currently the project manager of the FP7 ICT project HISTORIC on all optical logic and routing based on heterogeneous InP on silicon technology. 